\title{
ZDHHC8 as a candidate gene for schizophrenia: Analysis of a putative functional intronic marker in case-control and family-based association studies
} Thomas Faul ${ }^{1}$, Micha Gawlik ${ }^{1}$, Martin Bauer ${ }^{2}$, Sven Jung²,
Bruno Pfuhlmann'1, Burkhard Jabs², Michael Knapp ${ }^{3}$ and Gerald Stöber*1

Address: ${ }^{1}$ Department of Psychiatry and Psychotherapy, University of Würzburg, Füchsleinstraße 15, 97080 Würzburg, Germany, ${ }^{2}$ Department of Forensic Medicine, University of Würzburg, Lindleinstraße 15, 97080 Würzburg, Germany and 'Institute of Medical Biometry, Informatics and Epidemiology, University of Bonn, Sigmund-Freud-Str. 25, 53105 Bonn, Germany

Email: Thomas Faul - thomas.faul@gmx.de; Micha Gawlik - gawlik_m@klinik.uni-wuerzburg.de; Martin Bauer - reme005@mail.uniwuerzburg.de; Sven Jung - sven.jung@mail.uni-wuerzburg.de; Bruno Pfuhlmann - pfuhlmann_b@klinik.uni-wuerzburg.de; Burkhard Jabs - burkhard.jabs@mail.uni-wuerzburg.de; Michael Knapp - knapp@uni-bonn.de; Gerald Stöber* - stoeber_g@klinik.uniwuerzburg.de

* Corresponding author

Published: 14 October 2005

BMC Psychiatry 2005, 5:35 doi:10.1 186/147I-244X-5-35
Received: 15 July 2005

Accepted: 14 October 2005

This article is available from: http://www.biomedcentral.com/ I47I-244X/5/35

(C) 2005 Faul et al; licensee BioMed Central Ltd.

This is an Open Access article distributed under the terms of the Creative Commons Attribution License (http://creativecommons.org/licenses/by/2.0), which permits unrestricted use, distribution, and reproduction in any medium, provided the original work is properly cited.

\begin{abstract}
Background: The chromosome $22 \mathrm{qll}$ region is proposed as a major candidate locus for susceptibility genes to schizophrenia. Recently, the gene ZDHHC8 encoding a putative palmitoyltransferase at $22 \mathrm{qI}$ I was proposed to increase liability to schizophrenia based on both animal models and human association studies by significant over-transmission of allele rs I75 I74A in female, but not male subjects with schizophrenia.
\end{abstract}

Methods: Given the genetic complexity of schizophrenia and the potential genetic heterogeneity in different populations, we examined rs 75 I74 in 204 German proband-parent triads and in an independent case-control study (schizophrenic cases: $n=433$; controls: $n=186$ ).

Results: In the triads heterozygous parents transmitted allele $G$ preferentially to females, and allele A to males (heterogeneity $\chi^{2}=4.43 ; p=0.035$ ). The case-control sample provided no further evidence for overall or gender-specific effects regarding allele and genotype frequency distributions.

Conclusion: The findings on rsI75I74 at ZDHHC8 are still far from being conclusive, but evidence for sexual dimorphism is moderate, and our data do not support a significant genetic contribution of rsI75I74 to the aetiopathogenesis of schizophrenia.

\section{Background}

Systematic approaches designed to identify schizophrenia susceptibility genes have implicated the chromosome $22 \mathrm{q} 11$ microdeletion region as a promising hot-spot $[1,2]$. Recently, Mukai and colleagues [3] proposed a gender-specific effect of the ZDHHC8 gene at 22q11 to liabil- ity to schizophrenia based on animal models followed by human association studies. ZDHHC8 is a brain expressed putative palmitoyltransferase of 765 amino acids and may, thus, be involved in synaptic transmission and posttranslational modification of yet unidentified substrates. The ZDHHC8 gene consists of 11 exons spanning 16 kb 
on genomic DNA (http://www.genome.ucsc.edu/;

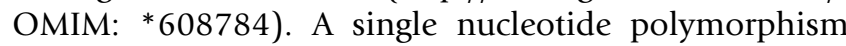
(SNP), rs175174 at intron 4, provided the highest significance from the entire 22q11 locus [4], and cell experiments gave evidence that rs 175174 (A/G) may modify ZDHHC8 expression by causing imperfect splicing, intron retention and reduced enzyme activity. Zdhhc8 knockout mice had sexually dimorphic deficits in prepulse inhibition and other features proposed to mimic schizophrenia in mice. Subsequently, fitting into the animal model analysis of 389 proband-parent trios showed significant overtransmission of allele A in female, but not male subjects with schizophrenia [3]. Studies aiming replication of the genetic evidence challenged the initial findings in populations of Asian or European origin either by a lack of association or by reporting the allele $\mathrm{G}$ over-transmitted to schizophrenia in a non-gender specific manner [5-7]. Given the genetic complexity of schizophrenia and the potential genetic heterogeneity in different populations as highlighted by the recent findings, we decided to conduct an association study on rs175174 in ZDHHC8 using both population- and family-based samples of an ethnically homogenous German population.

\section{Methods}

The proband-parent trio sample encompassed 204 cases, who fulfilled diagnostic criteria of DSM IV for schizophrenia [8], and their biological parents. The index cases (136 males; 67\%) had a mean age at onset of 23.8 years (SD 7.5 ) and an age at assessment of 32.4 years (SD 8.8). In 60 triads (29\%) we found a parent (33 females) previously treated for psychosis. There was no evidence for bilineal transmission in the sample. The sample for the independent case-control association study involved 433 probands with schizophrenia (321 males; $74 \%$ ). The probands had a mean age of first hospitalization at 28.9 years (SD 10.6), and an age at assessment of 39.2 (SD 13.5) years. In 15\% $(n=64)$ a further first-degree relative had been recorded as hospitalized for schizophrenia (familial cases). Subjects fulfilled diagnosis of cycloid psychoses $(n=149)$, unsystematic $(\mathrm{n}=155)$ and systematic schizophrenia $(\mathrm{n}=$ 129) according to differentiated psychopathology [9]. The probands were recruited at the Department of Psychiatry and Psychotherapy at the University of Würzburg. The 186 volunteer control subjects (105 males; 56\%) were recruited from the blood donor centre at the University of Würzburg at a mean age of 30.0 years (SD 10.3). All subjects were unrelated and of German Caucasian descent. The Ethics Committee of the University of Würzburg had approved the study, and informed consent was obtained from all subjects.

PCR for allelic discrimination at SNP rs175174 (A/G) was performed in a final reaction volume of $10 \mu \mathrm{l}$ containing 20 ng genomic DNA and $5 \mu$ lof $20 \times$ TaqMan ${ }^{\oplus}$ Universal
PCR Master Mix (Applied Biosystems) and $0.5 \mu \mathrm{l}$ of 20× $\mathrm{TaqMan}^{\mathrm{TM}}$ validated SNP genotyping assay including fluorescent tags specific for the wild type allele and the variant allele. Marker amplification was performed in microtiter plates on Biometra machines (Whatman). PCR amplification conditions were according to the facturer's recommendation $\left[10 \mathrm{~min}\right.$ at $95^{\circ} \mathrm{C}$ followed by $15 \mathrm{sec}$ at $92^{\circ} \mathrm{C}$ and $60 \mathrm{sec}$ at $60^{\circ} \mathrm{C}$ for 40 cycles]. Allelic discrimination with endpoint detection of fluorescence was performed at $60^{\circ} \mathrm{C}$ on an ABI prism 7000 sequence detection system followed by analysis with an appropriate software package (Applied Biosystems).

Fisher's exact and Armitage's trend test and were used to compare allelic and genotypic distributions between cases and controls. One-way ANOVA as implemented in the SAS was applied to compare the age-at-onset between genotypes. The data of the proband-parent triads were analyzed by the transmission-disequilibrium-test (TDT) [10]. Power calculations are based on the approximation described by Jackson et al. [11]; the exact test proposed by Weir [12] was applied for Hardy-Weinberg equilibrium.

\section{Results}

In the sample of 204 triads, no transmission distortion was apparent in the whole sample (TDT $=0.13, p=0.72$; Table 1 ) and in the sub-samples of families with maternal affection (TDT $=0.13, p=0.72$ ) or paternal affection (TDT $=0.17, p=0.68)$. Even after stratifying for gender of the proband, there was no significant over-transmission of an allele to males (TDT $=2.31, p=0.13$ ) and to females (TDT $=2.25, p=0.14$; Table 1). However, allele A is preferentially transmitted to males, whereas allele $\mathrm{G}$ is more often transmitted to females and this gender-related heterogeneity of allele transmission was significant (heterogeneity $\left.\chi^{2}=4.43 ; p=0.035\right)$.

In our case-control panel of 619 individuals, allele and genotype frequencies were not significantly different between cases and controls, and there was no evidence for gender-specific effects (Table 2). The familial/sporadic distinction produced negative results as did a sub-classification according to differentiated psychopathology (data not shown). Finally, genotypes were not associated with different mean age-at-onset in both panels (ANOVA p = 0.066). Genotypes were in appropriate Hardy-Weinberg equilibrium (HWE), and the allele frequency of rs $175174 \mathrm{G}$ was equal in the volunteer control sample (0.63) and the non-transmitted alleles in triads $(0.63)$.

\section{Discussion}

In independent case-control and proband-parent samples we attempted replication of a gender-specific transmission of allele rs $175174 \mathrm{~A}$ to females at the ZDHHC8 gene locus. In contrast to the original report of Mukai and 
Table I: Transmission of marker rs 175 I 74 A/G in 204 traids with schizophrenic psychoses

\begin{tabular}{|c|c|c|c|c|c|c|c|c|c|}
\hline \multirow[b]{2}{*}{ MT } & \multicolumn{3}{|c|}{ total sample $(\mathrm{N}=204)$} & \multicolumn{3}{|c|}{ females $(N=68)$} & \multicolumn{3}{|c|}{ males $(\mathrm{N}=136)$} \\
\hline & AA & AG & GG & AA & AG & GG & $\mathrm{AA}$ & AG & GG \\
\hline $\mathrm{AAxAA}$ & 32 & - & - & 10 & - & - & 22 & - & - \\
\hline$A A x A G$ & 37 & 31 & - & 9 & 13 & - & 28 & 18 & - \\
\hline AAxGG & - & 32 & - & - & 13 & - & - & 19 & - \\
\hline AGxAG & 14 & 23 & 14 & 4 & 7 & 8 & 10 & 16 & 6 \\
\hline AGxGG & - & 9 & 10 & - & 2 & 2 & - & 7 & 8 \\
\hline GGxGG & - & - & 2 & - & - & 0 & - & - & 2 \\
\hline $\mathrm{n}$ & 83 & 95 & 26 & 23 & 35 & 10 & 60 & 60 & 16 \\
\hline$\%$ & 40.7 & 46.6 & 12.7 & 33.8 & 51.5 & 14.7 & 44.1 & 44.1 & 11.8 \\
\hline HWE p-value & \multicolumn{3}{|c|}{0.37} & \multicolumn{3}{|c|}{0.78} & \multicolumn{3}{|c|}{0.37} \\
\hline TDT & \multicolumn{3}{|c|}{97 T/92 NT } & \multicolumn{3}{|c|}{$26 \mathrm{~T} / 38 \mathrm{NT}$} & \multicolumn{3}{|c|}{$7 / \mathrm{T} / 54 \mathrm{NT}$} \\
\hline $\mathrm{p}$-value & \multicolumn{3}{|c|}{0.72} & \multicolumn{3}{|c|}{0.13} & \multicolumn{3}{|c|}{0.13} \\
\hline
\end{tabular}

Transmission from heterozygous parents: $M T$ = Mating Type; HWE = Hardy-Weinberg-Equilibrium; TDT = transmission-disquilibrium test; $T=$ transmitted, NT = Non-transmitted.

Table 2: Allele and genotype frequency at marker rs 175 I 74 in schizophrenic psychoses

\begin{tabular}{|c|c|c|c|c|c|c|c|}
\hline \multirow[b]{2}{*}{ Samples } & \multicolumn{2}{|c|}{ Allele (\%) } & \multicolumn{5}{|c|}{ Genotypes (\%) } \\
\hline & $A$ & G & $\mathrm{P}$-value & AA & AG & GG & $\mathrm{P}$-value \\
\hline \multicolumn{8}{|l|}{ total } \\
\hline $\operatorname{SCZ}(N=433)$ & $527(60.9)$ & $339(39.1)$ & 0.48 & $154(35.6)$ & $219(50.6)$ & $60(13.9)$ & 0.43 \\
\hline $\operatorname{CON}(N=186)$ & $235(63.2)$ & $137(36.8)$ & & $71(38.2)$ & $93(50.0)$ & $22(11.8)$ & \\
\hline \multicolumn{8}{|l|}{ females } \\
\hline $\operatorname{SCZ}(N=112)$ & $125(55.8)$ & 99 (44.2) & 0.25 & $30(26.8)$ & $65(58.0)$ & $17(15.2)$ & 0.20 \\
\hline $\operatorname{CON}(N=8 I)$ & $100(61.7)$ & $62(38.3)$ & & $27(33.3)$ & $46(56.8)$ & $8(9.9)$ & \\
\hline \multicolumn{8}{|l|}{ males } \\
\hline $\operatorname{SCZ}(N=32 I)$ & $402(62.6)$ & $240(37.4)$ & 0.68 & $124(38.6)$ & $154(48.0)$ & $43(13.4)$ & 0.66 \\
\hline $\operatorname{CON}(N=105)$ & $135(64.3)$ & $75(35.7)$ & & $44(41.9)$ & $47(44.8)$ & $14(13.3)$ & \\
\hline \multicolumn{8}{|l|}{ family history } \\
\hline sporadic SCZ $(N=369)$ & $44 \mid(59.8)$ & $297(40.2)$ & 0.12 & $124(33.6)$ & $193(52.3)$ & $52(14.1)$ & 0.10 \\
\hline familial SCZ (N = 64) & $86(67.2)$ & $42(32.8)$ & & $30(46.9)$ & $26(40.6)$ & $8(12.5)$ & \\
\hline
\end{tabular}

SCZ: probands with schizophrenia; CON: controls; p-values for alleles (Fisher's exact test) and genotypes (Armitage's trend test).

colleagues [3] we found moderate evidence at p-level 0.035 of a sex-related heterogeneity of allele transmission with preferential transmission of allele A to males and allele $\mathrm{G}$ to females. This observation remained the only signal for a genetic involvement of ZDHHC8 in schizophrenia from our samples. The preferential transmission of allele $G$ corresponded partially to the report of Chen and colleagues [5] that allele G instead of A is preferentially transmitted to schizophrenic subjects, in opposite to the animal model and the initial genetic data from U.S. and Afrikaner pedigrees [3]. However, in the present study gender-specific effects were restricted to proband-parent triads, whereas one further study found non-gender related associations in both family-based and case-control samples [5] and two studies from different ethnic background were clearly negative in case-control [6] and both case-control and family-based designs [7]. Within European populations frequencies of allele $\mathrm{G}$ are consistent at 0.61 [7] and of 0.63 [present study], whereas in Asian populations allele $\mathrm{G}$ was the minor allele with 0.37 and $0.40[5,6]$. However, these differences hardly explain the contradictory genetic results, and assuming polygenic inheritance in schizophrenia, the negative findings could be only partially explained by sample stratification. 
To replicate weak gender-specific effects at rs175174 the study sample was relatively small, but the case-control sample possesses a power of $80 \%$ to detect (at $\alpha=0.05$ ) an association with a susceptibility allele, under the assumption that the susceptibility allele has a population frequency of 0.63 , and the effect of this allele is recessive with a relative risk of 2.5 . Assuming a multiplicative model, the effect size of the female replication sample of Mukai et al. [3] is 1.6 [7]. The power against this alternative was $57 \%$ for our case-control sample of females.

The inconclusive results obtained in several genetic studies raise the question whether SNP rs175174 is itself the susceptibility allele or is in linkage disequilibrium (LD) with a further functional variant that increases risk for schizophrenia. Whereas Saito et al. [6] proposed that rs 175174 is representative of ZDHHC8, differences in LD structure between the different populations may account for that the same disease causative variants yet to be identified are associated with different haplotypes. The functional variants could locate within ZDHHC8 or other genes around this locus at 22q11 and may independently or synergistically exert increased risk for schizophrenia. Therefore, the preferential transmission of individual alleles in at least a proportion of samples suggests the existence of additional functional variants in LD with SNP rs 175174 or an interaction effect on the risk haplotype. In addition, the effect of the non-spliced product bearing a premature termination codon on cell homeostasis and function is still unknown [3]. On the basis of these inconsistent genetic findings on rs 175174, the potential genetic diversity of the ZDHHC8 locus and the exact functional effect of related SNPs require further examination.

In conclusion, the findings at rs175174 at ZDHHC8 are still far from being conclusive, but evidence for a sexual dimorphism -if any- is weak. Given the large sample sizes studied together with the failure to replicate the initial findings in key respects, we agree with others [2] that the balance of evidence for $\mathrm{ZDHHCH} 8$ still favours the null hypothesis.

\section{Competing interests}

The author(s) declare that they have no competing interests.

\section{Authors' contributions}

TF carried out the molecular genetic studies and drafting of the manuscript, MG, SJ performed laboratory assays, $\mathrm{MB}$ participated in the design of the study and its coordination, $\mathrm{BP}, \mathrm{BJ}$ participated in the recruitment of the study sample, MK performed the data-analysis, interpretation of the data, and drafting of the manuscript, GS participated in the design and coordination of the study, interpretation of the data, and drafting of the manuscript. All authors read and approved the final manuscript.

\section{References}

I. Karayiorgou M, Gogos JA: The molecular genetics of the 22q I Iassociated schizophrenia. Brain Res Mol Brain Res 2004, 132(20):95- 104.

2. Craddock N, O'Donovan MC, Owen MJ: The genetics of schizophrenia and bipolar disorder: dissecting psychosis. J Med Genet 2005, 42:193-204.

3. Mukai J, Liu H, Burt R, Swor DE, Lai W-S, Karayiorgou M, Gogos JA: Evidence that the gene encoding ZDHHC8 contributes to the risk of schizophrenia. Nat Genet 2004, 36:725-73I.

4. Liu H, Abecasis GR, Heath SC, Knowles A, Demars S, Chen YJ, Roos JL, Rapoport JL, Gogos JA, Karayiorgou M: Genetic variation in the 22q I I locus and susceptibility to schizophrenia. Proc Natl Acad Sci USA 2002, 99:16859-16864.

5. Chen WY, Shi YY, Zheng YL, Zhao XZ, Zhang GJ, Chen SQ, Yang PD, $\mathrm{He} \mathrm{L}$ : Case-control study and transmission disequilibrium test provide consistent evidence for association between schizophrenia and genetic variation in the $22 \mathrm{qII}$ gene ZDHHC8. Hum Mol Genet 2004, I 3:299 I-2995.

6. Saito S, lkeda M, Iwata N, Suzuki T, Kitajima T, Yamanouchi Y, Kinoshita $Y$, Takahashi N, Inada T, Ozaki N: No association was found between a functional SNP in ZDHHC8 and schizophrenia in a Japanese case-control population. Neurosci Lett 2004, 374:2I-24.

7. Glaser B, Schumacher J, Williams HJ, Jamra RA, lanakiev N, Milev R, Ohlraun S, Schulze TG, Czerski PM, Hauser J, Jonsson EG, Sedvall GC, Klopp N, Illig T, Becker T, Propping P, Williams NM, Cichon S, Kirov G, Rietschel M, Murphy KC, O'Donovan MC, Nothen MM, Owen MJ: No association between the putative functional ZDHHC8 single nucleotide polymorphism rs 175174 and schizophrenia in large European samples. Biol Psychiatry 2005, 58:78-80.

8. American Psychiatric Association: Diagnostic and statistical manual of mental disorders. 4th ed. text revision. (DSM-IV-TR) Washington, DC: American Psychiatric Association; 2000.

9. Leonhard K: Classification of endogenous psychoses and their differentiated etiology. 2nd rev. and enlarged ed Wien, New York: Springer; 1999.

10. Schaid DJ, Sommer SS: Genotype relative risks: methods for design and analysis of candidate-gene association studies. Am J Hum Genet 1993, 53: I I 14-26.

11. Jackson MR, Genin E, Knapp M, Escary JL: Accurate power approximations for $\chi^{2}$-tests in case-control association studies of complex disease genes. Ann Hum Genet 2002, 66:307-32I.

12. Weir BS: Genetic data analysis II Sunderland, Massachusetts: Sinauer Associates; 1996.

\section{Pre-publication history}

The pre-publication history for this paper can be accessed here:

http://www.biomedcentral.com/1471-244X/5/35/pre pub 